\title{
ON THE MASS OF THE GRAVITATIONAL LENSES IN LMC
}

\author{
L. MANCINI* AND G. SCARPETTA ${ }^{\dagger}$ \\ Dipartimento di Fisica, Università di Salerno, I-84081 Baronissi (SA), Italy \\ S. CALCHI NOVATI ${ }^{\ddagger}$ AND PH. JETZER \\ Institut für Theoretische Physik der Universität Zürich, CH-8057 Zürich, Switzerland
}

\begin{abstract}
In the self-lensing framework, we estimate the modal values of the mass of the gravitational lenses found by the MACHO collaboration towards the Large Magellanic Cloud (LMC). Our results suggest that only the events located near the center can be identified as a low mass star population belonging to the LMC disk or bar components.
\end{abstract}

\section{Microlensing towards the Large Magellanic Cloud}

The microlensing surveys towards the Large Magellanic Cloud (LMC) have demonstrated the existence of compact objects that act as gravitational lenses somewhere between us and LMC. Besides the 4 events found by the EROS2 group [1], the MACHO Project has detected 16 events and its maximum likelihood estimate of the mass of the lensing objects is $\approx 0.5 \mathrm{M}_{\odot}$, while the fraction $f$ of dark matter in form of MACHOs in the Galactic halo is estimated to be $\sim 20 \%$ [2]. However, the interpretation of these data is a matter of controversy and other hypothesis have been proposed: the possibility that the lenses are located in LMC itself instead as in the Galactic halo was claimed by Sahu [3]; a warp of the Milky Way (MW) disk, which covers the line of sight towards LMC, has been proposed to support a lens disk population [4]; Zhao [5] has suggested instead that the debris torn from the LMC by tidal forces may be a source of MACHOs; a non planar geometry of LMC, i.e. a misalignment of the bar from the the disk, has been proposed by Zhao \& Evans [6]; other authors have considered LMC components fatter than is conventional, with material extending to scale heights of $\sim 6 \mathrm{kpc}$ above the plane of the LMC disk [7], as is suggested by Weinberg [8] numerical simulations of the evolution of the LMC in the tidal field of the MW. The analysis of Jetzer et al. [9] has shown that possibly the observed events are distributed among different galactic components (disk, spheroid, galactic halo, LMC halo and self-lensing). This means that the lenses do not belong all to the same population and their astrophysical features can

${ }^{*}$ L.M. is partially supported by the Swiss National Science Foundation.

${ }^{\dagger}$ G.S. is partially supported by the Istituto Nazionale di Fisica Nucleare, sez. Napoli, Italy.

${ }^{\ddagger}$ S.C.N. is supported by the Swiss National Science Foundation and the Tomalla Foundation. 
differ deeply one another. The microlensing surveys towards the Small Magellanic Cloud have detected very few events, which did not help to clarify the problem as it was hoped.

\section{A new picture of the Large Magellanic Cloud}

A new accurate description of the LMC geometry and dynamics was recently proposed by van der Marel et al. [10], thanks to the DENIS and 2MASS surveys. From their study emerges that the distribution of neutral gas is not a good tracer, and thus leads to an incorrect LMC model. Instead, using the carbon star data, they have provided an accurate measurement of the dynamical center of the stars, which turns out to be consistent with the center of the bar. Moreover, they have pointed out that the shape of the LMC disk is not circular, but elliptical. In this case, the position angle of the line of nodes is different from that of the major axis of the LMC disk. These facts change completely the previous geometry and a noticeable asymmetry between the two sides of LMC divided by the line of the nodes arises when studying the positions of the MACHO microlensing event towards LMC.

\section{Optical Depth and Asymmetry}

By using the van der Marel et al. description of LMC, we have reported new accurate estimates of the optical depth for any source/lens configuration [11]. Our results show an evident near-far asymmetry of the optical depth values for lenses located in the LMC halo. This asymmetry is due to the fact that the LMC disk is inclined so that line of sights towards the far side of LMC go through a larger portion of the LMC halo, according to the prediction of Gould [12]. The flaring of the LMC disk is a further source of the asymmetry of the optical depth between the far and the near side of LMC. The asymmetry is completely lost, as expected, in the self-lensing case.

We have also provided a set of statical calculations to study the asymmetry among the locations of the microlensing events found. Our results show that the existence of the near-far asymmetry supports the common idea that the LMC is surrounded by a halo of dark material [11].

\section{Modal mass estimates for lenses in LMC}

Evans and Kerins [7] have noticed that the distribution of timescales of the events and their spatial variation across the face of the LMC disk offers possibilities of identifying the dominant lens population. We find that the observed timescales distribution of the events found by the MACHO collaboration as a function of the distance from the center of LMC is not in agreement with that theoretically expected for lenses located all in the inner galactic components of LMC. Our statistical analysis allows to distinguish in principle at least two different lens populations. In particular, only the events concentrated near the center of LMC with self-lensing 
optical depth $\tau_{\mathrm{sl}}>2 \times 10^{-8}$, have a good chance to be true self-lensing events [11]. We have estimated the modal values of the masses of these lenses. The results suggest that they could belong to a low mass star population in the LMC disk/bar components. In Fig. 1 we show the scatter plot of the modal value of the lens mass $\mu$ with respect to the measured Einstein time for each MACHO event. Filled (empty) triangles represent points with $\tau_{\mathrm{sl}}>2 \times 10^{-8}\left(<2 \times 10^{-8}\right)$. The horizontal line indicates the lower limit of the lens mass for self lensing. Four MACHO events have a modal value of the lens mass smaller than the lower limit and therefore are not represented in the figure. We also excluded from our analysis the binary event [13] and the Galactic disk event [14]. The dashed line represents the correlation line for the 6 events with $\tau_{\mathrm{sl}}<2 \times 10^{-8}$. These events are located far from the LMC center and can hardly be identified as belonging to a LMC population.

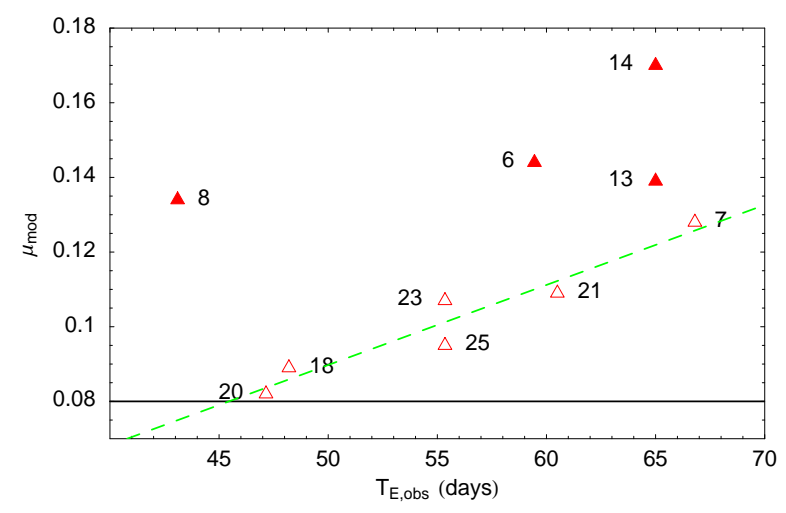

Figure 1. Scatter plot of the modal value of $\mu$ (y-axis) with respect to the measured Einstein time ( $x$-axis) of the event, where $\mu$ is the lens mass in solar mass units. The label identifies the MACHO event as reported in [2].

\section{References}

1. Lasserre T., Afonso C., Albert J.N. et al., A\&A 355, L39 (2000)

2. Alcock C., Allsman R.A., Alves D.R. et al., ApJ 542, 281 (2000)

3. Sahu K.C., PASP 106, 942 (1994)

4. Evans N.W., Gyuk G., Turner M.S. \& Binney J., ApJ 501, 45 (1998)

5. Zhao H.S., MNRAS 294, 139 (1998)

6. Zhao H.S. \& Evans N.W., ApJ 545, L35 (2000)

7. Evans N.W. \& Kerins E., ApJ 529, 917 (2000)

8. Weinberg M.D., ApJ 532, 922 (2000)

9. Jetzer Ph., Mancini L. \& Scarpetta G., A\&A 393, 129 (2002)

10. van der Marel R.P., Alves D.R., Hardy E. et al., AJ 124, 2639 (2002)

11. Mancini L., Calchi Novati S., Jetzer Ph. \& Scarpetta G. , A\&A in press (2004)

12. Gould A., ApJ 404, 451 (1993)

13. Alcock C., Allsman R.A., Alves D.R. et al., ApJ 541, 270 (2000)

14. Alcock C., Allsman R.A., Alves D.R. et al., Nature 414, 617 (2001) 